\title{
Visual Prompts will not Clear
}

National Cancer Institute

\section{Source}

National Cancer Institute. Visual Prompts will not Clear. NCI Thesaurus. Code C62861.

Problem with visual messages which continue to be displayed on/by the device after the appropriate action has been taken. 